\title{
Dielectric Coupler for General Purpose Q-Band EPR Cavity
}

\author{
Martyna Judd ${ }^{1} \cdot$ Greg Jolley $^{1} \cdot$ Dieter Suter $^{2} \cdot$ Nicholas Cox $^{1} \cdot$ Anton Savitsky $^{2}$ (D)
}

Received: 31 May 2021 / Revised: 20 July 2021 / Accepted: 27 July 2021 /

Published online: 19 August 2021

(c) The Author(s) 2021

\begin{abstract}
Here, we report on a robust and efficient mechanism for tuning the microwave coupling of a Q-band (34 GHz), general purpose, cylindrical EPR cavity operating in the $\mathrm{TE}_{011}$ mode. This novel mechanism allows for both the adjustment of the cavity's coupling over a wide frequency range, as well as its bandwidth from that of a high-Q cavity (about $10 \mathrm{MHz}$ ), to a broadband cavity (above $1 \mathrm{GHz}$ ). The coupling element consists of a dielectric plate fixed onto a movable waveguide short that allows for two modes of operation. In the first mode, the dielectric plate does not influence the resonance properties of the coupling iris and allows for precise, critical coupling of the high-Q cavity. In the second mode, the dielectric plate is positioned in front of the coupling iris, varying the iris' resonance properties and allowing very strong overcoupling to be achieved. This mechanism can be generalized for other types of EPR cavities, in particular at high microwave frequencies.
\end{abstract}

\section{Introduction}

Owing to sensitivity requirements, EPR measurements are almost always performed using a resonator, where the properties of the resonator are tuned for the particular type of EPR experiment. In continuous wave (CW) EPR measurements low-loss (high-Q) resonators are used, as they efficiently concentrate the available microwave magnetic field at the sample, enhancing sensitivity. As such, resonators used in classical CW EPR applications typically only have a small resonator bandwidth. In contrast, pulse EPR measurements require a sufficiently large resonator bandwidth. Many pulse EPR measurements drive off-resonance transitions, which can only be efficiency pumped if they fall within the resonator bandwidth, regardless of the

Anton Savitsky

anton.savitsky@tu-dortmund.de

1 Research School of Chemistry, The Australian National University, 137 Sullivans Creek Rd, Acton, Canberra ACT 2601, Australia

2 Faculty of Physics, Technical University Dortmund, Otto-Hahn-Str. 4a, 44227 Dortmund, Germany 
bandwidth of the source or the duration of the microwave pulse(s). How bandwidth limitations affect pulse EPR experiments (ESEEM, ENDOR, DEER, EDNMR, etc.) is described in detail in the supplementary information (SI), see also [1, 2]. In addition, the resonator bandwidth places restrictions on the pulse lengths and timings. This includes the minimum pulse length that can be achieved without distortion, and in many circumstances, the dead time of the pulse experiment, with the dead time being the delay between the applied microwave pulse (excitation power in the range of Watts to kilowatts) and subsequent signal detection (signal power in the nanoWatt range). As such, resonators used for pulse EPR ideally need large bandwidths. Current EPR technology routinely allows minimum microwave pulse lengths of the order of $10 \mathrm{~ns}$ (excitation bandwidth corresponds to $120 \mathrm{MHz}$ [3]). Consequently, the ideal resonator bandwidth needs to be at least $\pm 100 \mathrm{MHz}$, i.e., $200 \mathrm{MHz}$, regardless of the operating frequency.

The mismatch between the resonator requirements needed for $\mathrm{CW}$ and pulse EPR measurements makes the construction of a general purpose resonator challenging. This problem has been overcome at low frequencies $(<10 \mathrm{GHz})$ by developing mechanisms to vary the coupling of the EPR resonator to the microwave source via an intervening transmission line. Such coupling mechanisms allow for two modes of operation: (i) a high-Q regime for $\mathrm{CW}$ measurements in which the resonator is 'critically coupled' to the transmission line; and (ii) a broad bandwidth regime for pulse EPR in which the resonator is described as 'overcoupled'; see supplementary information. There are many different types of coupling mechanisms (couplers). A key criterion of the coupler is the range of coupling strengths it allows. In CW EPR, the coupling range determines the maximum sample size of samples with high dielectric losses. In pulse EPR experiments, it limits the maximum resonator bandwidth. At low microwave frequencies such as X-band $(9.5 \mathrm{GHz})$, dielectric or loop-gap resonators, which are typically coupled by an antenna coupling mechanism, perform well in both regimes.

At higher microwave frequencies, i.e., Q-band $(34 \mathrm{GHz})$ and above, resonators are typically connected to the source via a hollow rectangular waveguide. Microwaves pass from the rectangular waveguide to the resonator through a thin metal diaphragm, which accommodates an aperture of fixed geometry, i.e., a slot or circular iris. The variability of the coupling is achieved using several approaches which regulate the transmittance of the iris and thus the cavity coupling (see SI). Cylindrical cavities, operating in the $\mathrm{TE}_{011}$ mode, typically use a compact sliding short in the waveguide to couple the transmission line and resonator. This design is suitable for CW and pulsed EPR experiments and is realized in several commercially available (Bruker Biospin) and laboratory-developed EPR probeheads [4-6]. One example is the general purpose Q-band probehead developed at the TU Berlin, which is now used in several EPR laboratories worldwide [5]. The original probehead allowed CW EPR and a large variety of pulsed EPR experiments including ENDOR, but had limited ESEEM, DEER and EDNMR performance because the maximum bandwidth that can be achived by the short coupler is limited to $<50 \mathrm{MHz}$ [5].

In this work, we describe an improved short coupler design that overcomes this limitation. The new probehead design allows for precise, critical coupling of high-Q cavities and for very strong overcoupling, yielding a cavity bandwidth $>200 \mathrm{MHz}$, 
ideal for pulse EPR experiments. Strong overcoupling is achieved by modifying the iris self-resonance properties using a dielectric material. In addition, it is shown that the performance of the resonator-coupler-transmission line unit can be accurately predicted by electromagnetic calculations, allowing future development.

\section{Materials and Methods}

\subsection{Microwave Resonator}

The probehead model used for the electromagnetic calculations is depicted in Fig. 2a. The dimensions of the model correspond to those of the cavity used in the experiments (Table 1) and are also very similar to the dimensions of general purpose Q-band resonators described previously [5]. Dielectric plates were prepared from laminate Rogers 3010 materials. Pieces with thicknesses of 100, 210, 250 or $290 \mu \mathrm{m}$ were prepared. The material was affixed to the resonator using ethyl 2-cyanoacrylate adhesive.

\subsection{Electromagnetic Simulations}

These were performed using the CST microwave studio package. The reflection $\left(S_{11}\right)$ and transmission $\left(S_{21}\right)$ coefficients, and the electric and magnetic field amplitudes were calculated using a frequency domain solver with a tetrahedral mesh adjusted to yield reproducible results (typically about $10^{6}$ tetrahedrons for the full cavity model). The transmission properties of the isolated iris were calculated using an aperture of different geometries (round, slot etc.) in a WR28 waveguide. Losses were neglected in the simulations of the isolated iris, i.e., a perfect conductor and lossless dielectric material were assumed.

Table 1 Summary of final probehead model geometry and materials

\begin{tabular}{|c|c|c|}
\hline & Dimensions & Material \\
\hline Cavity & $\begin{array}{l}\text { Diameter: } 10.9 \mathrm{~mm} \\
\text { Height: } 9.2 \mathrm{~mm}^{\mathrm{b}} \\
\text { Iris wall thickness: } 0.1 \mathrm{~mm}\end{array}$ & Brass, $\sigma=1.6 \cdot 10^{7} \mathrm{~S} / \mathrm{m}^{\mathrm{c}}$ \\
\hline ENDOR posts $^{\mathrm{a}}$ & Diameter: $0.8 \mathrm{~mm}$ & Silver, $\sigma=6.3 \cdot 10^{7} \mathrm{~S} / \mathrm{m}$ \\
\hline Sample tube & $\mathrm{OD}=2.8 \mathrm{~mm}, \mathrm{ID}=1.8 \mathrm{~mm}$ & Quartz, $\varepsilon_{\mathrm{r}}=3.75, \tan \delta=4 \cdot 10^{-4}$ \\
\hline Dielectric plate & $\begin{array}{l}\text { Width: } 3.0 \mathrm{~mm} \\
\text { Height: } 10 \mathrm{~mm} \\
\text { Thickness: } D T \text {-variable }\end{array}$ & $\begin{array}{l}\text { Rogers RT3010, } \\
\quad \varepsilon_{\mathrm{r}}=10.7^{\mathrm{d}} ; \\
\tan \delta=2.3 \cdot 10^{-3} \text { at } 10 \mathrm{GHz}\end{array}$ \\
\hline
\end{tabular}

${ }^{\text {a}}$ Positioned as described in [5]

${ }^{\mathrm{b}}$ For a resonance frequency of $34 \mathrm{GHz}$ in the $\mathrm{TE}_{011}$ mode

${ }^{\mathrm{c}}$ The material conductivity was adjusted to match the calculated and experimental $Q_{U}$-values

${ }^{\mathrm{d}}$ The average between process $(10.2 \pm 0.3)$ and design (11.2) values specified by Rogers at $10 \mathrm{GHz}$ 


\subsection{Q-Band mw Bridge Extension}

A home-built X-to-Q-band bridge extension compatible with a Bruker ELEXSYS-I $\mathrm{X}$-band EPR spectrometer was used for all experiments. A simplified block diagram of the bridge extension is shown in Fig. 1. Further details are described in the supplementary information.

\subsection{EPR Measurements}

1,3-Bis(diphenylene)-2-phenylallyl (BDPA) radical (Sigma Aldrich) prepared in ortho-terphenyl (Sigma Aldrich) matrix (0.1\% BDPA concentration) was used for all experiments.

The bandwidth of the critically coupled and overcoupled resonator was estimated by performing microwave nutation measurements across the full frequency range of the microwave bridge $(33.5-34.5 \mathrm{GHz})$. The pulse sequence used for the nutation experiment was $t_{\mathrm{p}}-T-t_{\pi / 2}-\tau-2 t_{\mathrm{p} \pi / 2}-\tau-e c h o$. The $\pi / 2$ pulse length, $t_{\pi / 2}$, was fixed to $40 \mathrm{~ns}, \tau$ was set to $1000 \mathrm{~ns}$ and the delay between the preparative pulse $t_{\mathrm{p}}$ and echo detection was fixed at $T=10 \mu \mathrm{s}$. The length of the preparative pulse $t_{\mathrm{p}}$ was incremented in $4 \mathrm{~ns}$ steps. The static magnetic field for different microwave frequencies was adjusted to maintain the resonance condition, i.e., achieve the maximum spin echo intensity at each frequency offset. At room temperature a shot repetition time of $5 \mathrm{~ms}$ was used. At low temperatures (20-50 K), the shot repetition time was extended to $400 \mathrm{~ms}$. The resonator ringdown decay was measured using an external oscilloscope, by recording the response of the system following a low power pulse of $200 \mathrm{~ns}$.

The procedure used to reproducibly overcouple the resonator was as follows: the lower and higher critically coupled short positions were identified, as visualized using the spectrometer's in-built voltage controlled oscillator (VCO, i.e., tune mode). The short was then set at the position halfway between. At room

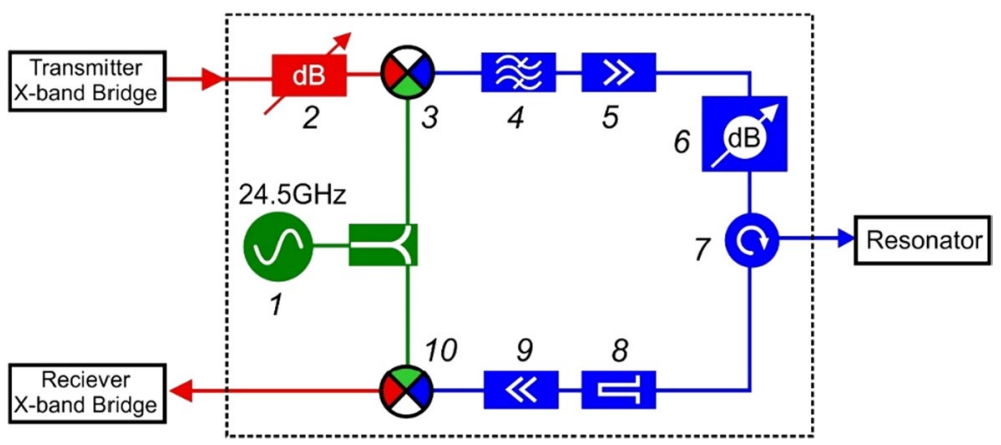

Fig. 1 Basic scheme of the the pulse Q-band bridge extension: (1) DRO-based fixed frequency mw source; (2) voltage controlled attenuator; (3) up-converter mixer; (4) bandpass filter; (5) high-power mw amplifier; (6) rotary vane attenuator; (7) circulator; (8) fast PIN switch; (9) low-noise amplifier; (10) down-converter mixer. Full schematic details including power output measurements are presented in the SI 
temperature, the maximally overcoupled short position was further optimized using water as a microwave absorber, as described previously [7]. The sample tube was loaded with two thin-wall capillaries $(0.8 \mathrm{~mm} \mathrm{OD}, 0.6 \mathrm{~mm}$ ID) filled with water, offset from the bottom of the tube by a $5 \mathrm{~mm}$ length of quartz or cotton wool. The water sample was inserted into the resonator to the maximum length where it could still be critically coupled. The short position at which critical coupling for water is achieved corresponds to the maximally overcoupled position for the BDPA sample.

\section{Results}

\subsection{Electromagnetic Simulations}

Figure 2 shows the electromagnetic calculation results for the cavity model with a circular iris of $2.6 \mathrm{~mm}$ diameter. The cavity height was adjusted such that the $\mathrm{TE}_{011}$ cavity mode appeared at $34 \mathrm{GHz}$. In the $28 \mathrm{GHz}-40 \mathrm{GHz}$ frequency range, only two additional modes can be identified: a $\mathrm{TE}_{211}$ mode at about $30.18 \mathrm{GHz}$, and a $\mathrm{TE}_{311}$ mode at $39.44 \mathrm{GHz}$ (Fig. 2b). Additional eigenmode analysis reveals that all transverse magnetic modes $\left(\mathrm{TM}_{012}, \mathrm{TM}_{110}, \mathrm{TM}_{111}\right)$ in this range are suppressed by the four ENDOR posts located symmetrically about the sample tube. Also note that the transverse electric $\mathrm{TE}_{112}$ mode and additional hybrid modes caused by the ENDOR posts and the sample tube are not excited by the sidewall iris. This is not the case for top/bottom wall irises [8] and represents a key advantage of the sidewall iris design.
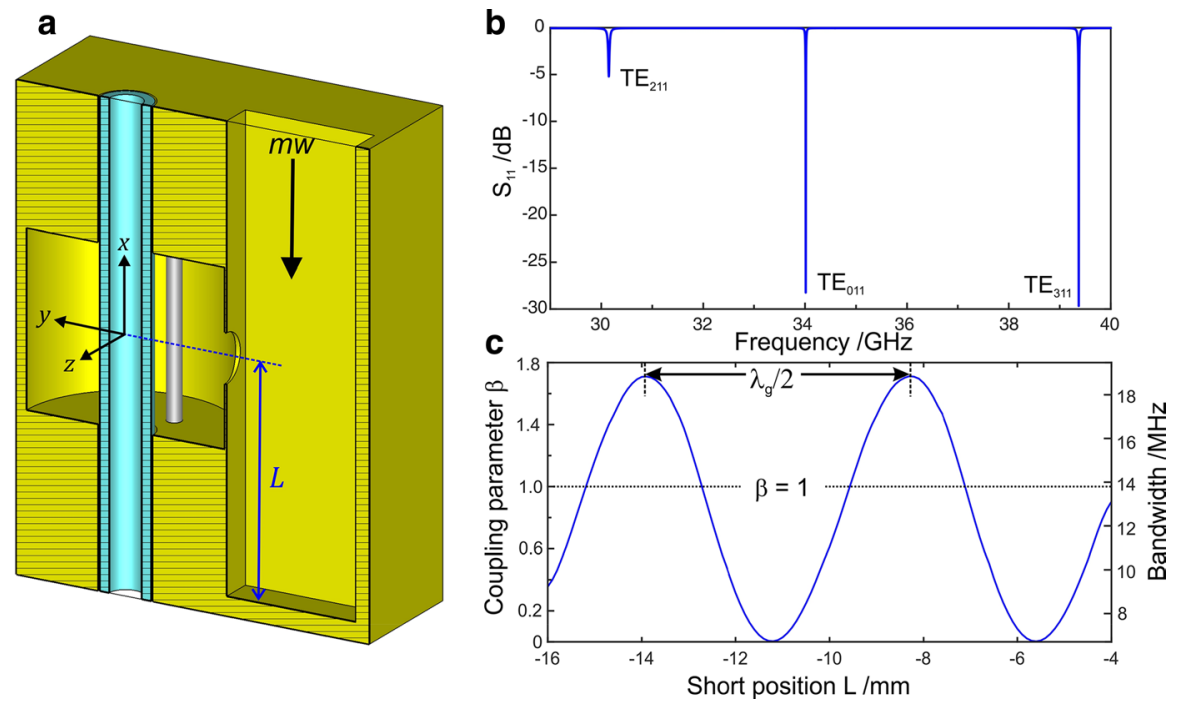

Fig. 2 a Model of the $\mathrm{TE}_{011}$ cavity used for electromagnetic simulations. The geometrical and material parameters of the model are summarized in Table 1 . The coordinate system used in this work is annotated in the figure. b Reflection coefficient, $\mathrm{S}_{11}$, for a critically coupled cavity (short position $L=-$ $9.7 \mathrm{~mm}$ ). c Dependence of the coupling coefficient (resonator bandwidth) on the short position relative to the center of a circular iris of $2.6 \mathrm{~mm}$ diameter 
Figure 2c shows the dependence of the coupling parameter $(\beta)$ and cavity bandwidth $\left(\Delta v_{1 / 2}\right)$ of the $\mathrm{TE}_{011}$ mode as a function of the short position, as evaluated from the reflection curves, $S_{11}$, for different short positions. The short position regulates the waveguide-to-cavity transmission coefficient of the iris which is related to cavity coupling; see SI. Maximum transmission and coupling is achieved for short positions where the transverse electric ( $z$-direction) and magnetic ( $x$-direction) field amplitudes are maximal at the iris, i.e., short positioned at $L(\beta=\max )=.n \frac{\lambda_{g}}{2}+\frac{\lambda_{g}}{4}$ $=(8.4 \mathrm{~mm}, 14.0 \mathrm{~mm}, \ldots)$ with $\lambda_{g}=11.24 \mathrm{~mm}$ being the wavelength of the WR2 8 waveguide at $34 \mathrm{GHz}$ and $n=0,1,2 \ldots$ Zero transmission (no coupling) takes place for the short positioned at $L(\beta=0)=n \frac{\lambda_{g}}{2}$ with respect to the iris center, i.e., zero field amplitudes at the iris center. Here we opted for an iris diameter of $2.6 \mathrm{~mm}$ of the experimental probehead. While suitable for CW-EPR, this iris diameter yields only a maximum bandwidth of $18.7 \mathrm{MHz}$ ( $\beta=1.7$, i.e., overcoupled), which is severely below the minimum bandwidth required for many pulse EPR experiments.

The diameter of the iris modulates the maximum waveguide-to-cavity transmittance of the iris, with a larger diameter resulting in a larger bandwidth; see Fig. S3 in the SI. The largest possible circular iris $(3.55 \mathrm{~mm})$ is constrained by the dimensions of the waveguide (WR28 waveguide dimensions of $7.116 \times 3.556 \mathrm{~mm}^{2}$ ). Using a $3.55 \mathrm{~mm}$ iris diameter yields a maximum cavity bandwidth of $100 \mathrm{MHz}$, but also reduces the unloaded $Q$-value, and thus the maximum magnetic field amplitude for the critically coupled cavity, which is disadvantageous for CW EPR; see Fig S3 in the SI.

To obtain a higher cavity bandwidth while keeping the iris diameter small, the iris transmission coefficient to the cavity must be increased. This is best realized by adjusting the iris self-resonance frequency to the resonance frequency of the $\mathrm{TE}_{011}$ mode, which will result in both the highest wave transmission and the maximum cavity bandwidth. Positioning a dielectric plate with a high dielectric constant in front of the iris increases the electric and magnetic field amplitudes at the iris. This is equivalent to increasing of the iris inductance and capacitance, and leads to a downshift in the iris resonance frequency [9]. Calculations which included a dielectric plate $(\varepsilon=10.7)$ of $3 \times 3 \mathrm{~mm}^{2}$ placed in front of the isolated iris of $2.6 \mathrm{~mm}$ diameter do indeed show a continuous downshift of the self-resonance frequency and a corresponding increase in the transmission coefficient at $34 \mathrm{GHz}$ as a function of the dielectric plate thickness. A maximum transmission coefficient of $T=0.96$ is predicted for a dielectric plate thickness of $0.32 \mathrm{~mm}$; see Fig S5 in the SI.

Figure $3 \mathrm{a}$ shows the reflection coefficient for a full cavity model calculated without a dielectric plate and the short position optimized for critical coupling. The frequency dependence of the magnetic field amplitude ( $x$-component) at the cavity center is shown in Fig. 3c. Analysis of both plots yields a cavity bandwidth of $\Delta v_{1 / 2}^{0}=13.7 \mathrm{MHz}, Q_{U}=2 \cdot \frac{v_{0}}{\Delta v_{1 / 2}^{0}}=4960$. Inspection of the magnetic field distribution at the resonance frequency shows the field distribution is very similar to that of the canonical $\mathrm{TE}_{011}$ mode, which is slightly distorted by the quartz tube and ENDOR posts (see inserts in Fig. 3c).

The corresponding case for a dielectric plate of DT $=0.25 \mathrm{~mm}$ and a short position optimized for maximum overcoupling (waveguide-cavity transmittance) is 

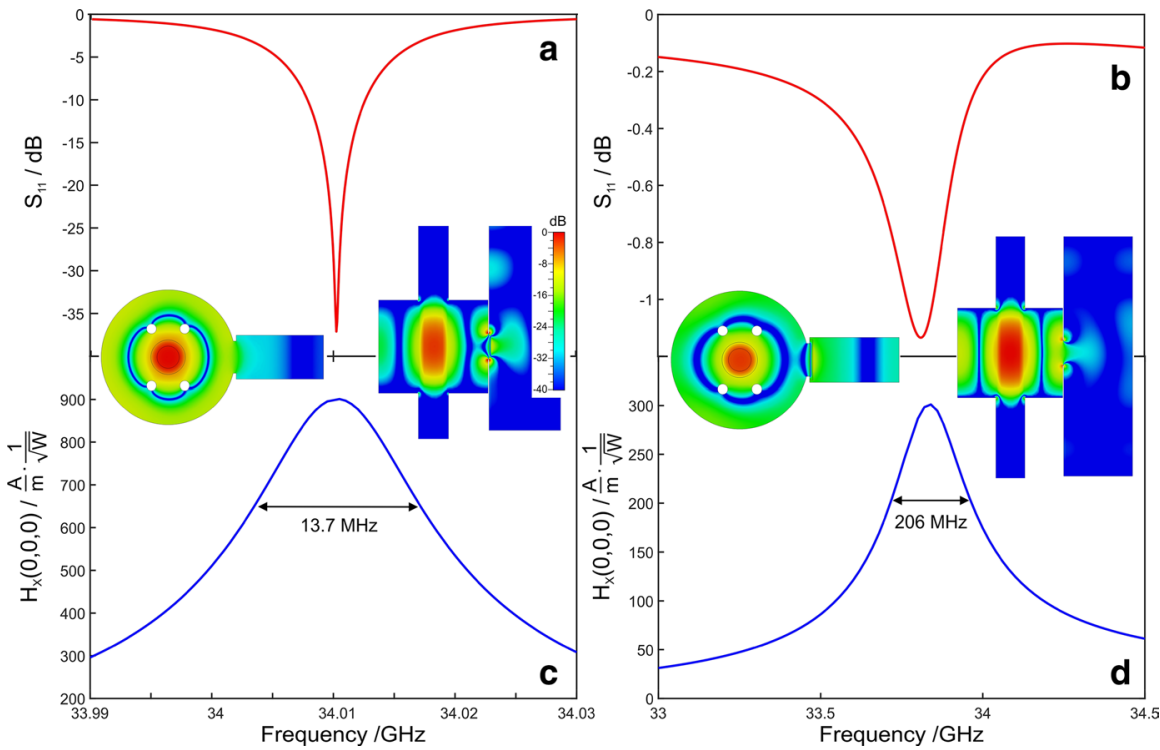

Fig. $3 \mathrm{~S}_{11}$ parameters of the a critically coupled $(\mathrm{DT}=0)$ and $\mathbf{b}$ max. overcoupled $(\mathrm{DT}=0.25 \mathrm{~mm}, 3 \times 3$ $\mathrm{mm}^{2}$, fixed on iris) resonator, with $2.6 \mathrm{~mm}$ iris diameter. $x$-component of the magnetic field amplitude, $H_{x}(0,0,0)$, at the resonator center for c critically coupled and $\mathbf{d}$ max. overcoupled resonator. The calculated normalized distributions of the magnetic field $x$-amplitude, $H_{x} / H_{x}(0,0,0)$, in the (left) $y, z$-plane $(x=0)$ and (right) $x, z$-plane $(y=0)$ are shown in inserts on a logarithmic scale

shown in Fig. 3 b, d. Both the $S_{11}$ curve and magnetic field amplitude curves have asymmetrical profiles. The magnetic field distribution in the cavity is distorted as compared to that in the model without a dielectric plate (see inserts Fig. 3d). A cavity bandwidth of $206 \mathrm{MHz}$ is evaluated from the frequency dependence of the magnetic field amplitude, Fig. 3d, which is already suitable for most pulse EPR applications. The maximum magnetic field amplitude is reduced by a factor of 2.9 compared to the critically coupled case without a dielectric plate $\left(310 \frac{\mathrm{A}}{\mathrm{m}} \cdot \frac{1}{\sqrt{\mathrm{W}}} v s\right.$. $\left.909 \frac{\mathrm{A}}{\mathrm{m}} \cdot \frac{1}{\sqrt{\mathrm{W}}}\right)$. This number is consistent with the theoretical value of 2.79 , which assumes a "true" overcoupling regime where an increase of the cavity bandwidth (coupling coefficient) occurs without change of the unloaded Q-value, i.e., field distributions within the cavity remain constant (see SI and [10]):

$$
\frac{H_{x}(0,0,0)}{H_{x}^{0}(0,0,0)}=\sqrt{2-\frac{\Delta v_{1 / 2}^{0}}{\Delta v_{1 / 2}}} \cdot \sqrt{\frac{\Delta v_{1 / 2}^{0}}{\Delta \nu_{1 / 2}}} .
$$

However, if the short is adjusted to the critical coupling position, a value of $\Delta v_{1 / 2}=26.4 \mathrm{MHz}$ and $H_{x}(0,0,0)=640 \frac{\mathrm{A}}{\mathrm{m}} \cdot \frac{1}{\sqrt{\mathrm{W}}}$ is obtained from both the $\mathrm{S}_{11}$ and $H_{x}$ frequency plots. This cavity bandwidth corresponds to $Q_{U}=2580$, which is about half the $Q_{U}$ without a dielectric plate, i.e., the power losses increase by a factor of 2. Additional losses in the system originate from changes in the magnetic 
field distribution, which cause an increase in current density at the cavity walls and iris, and dielectric losses in the dielectric plate. The additional losses from two sources are about equal, i.e., $Q_{\text {cond }}=10800$ and $Q_{\text {diel }}=10,700$ for materials used in the model. It is thus desirable to be able to introduce and remove the dielectric plate to optimize performance in both the critically coupled (CW-EPR) and overcoupled (pulse EPR) regime.

To understand the action of the dielectric plate in more detail, we calculated the dependence of the $S_{11}$ coefficient on the dielectric plate thickness. Figure $4 \mathrm{a}$ shows the reflection coefficients in the frequency range of $27-42 \mathrm{GHz}$, represented as contour plots. For thin dielectric plates, well-isolated $\mathrm{TE}_{211}, \mathrm{TE}_{011}$ and $\mathrm{TE}_{311}$ modes are observed. The self-resonance frequency of the iris is shown using a dashed line. It initially appears off-scale, decreasing as the thickness of the dielectric plate increases, approaching the resonance frequency of the $\mathrm{TE}_{011}$ mode $(34 \mathrm{GHz})$, at a thickness of $0.32 \mathrm{~mm}$. Simultaneously, the $\mathrm{TE}_{011}$ mode begins to couple with both the $\mathrm{TE}_{211}$ mode and particularly the $\mathrm{TE}_{311}$ mode to produce mixed TE-modes. This is readily observed in the calculated magnetic field magnitude distributions, with the $\mathrm{TE}_{011}$ mode gaining $\mathrm{TE}_{311}$ character (see Fig. S6 in SI). An interesting situation is established as the iris self-resonance approaches the frequency of $\mathrm{TE}_{011}$ mode. Typical mode passage is observed with the closest approach of the two modes at DT $=0.34 \mathrm{~mm}$, which is close to the $0.32 \mathrm{~mm}$ required for an isolated iris self-resonance frequency of $34 \mathrm{GHz}$.

From the perspective of optimizing EPR performance, the two significant parameters are the resonator bandwidth and the amplitude of the microwave magnetic field coincident with the sample, and thus only these parameters were
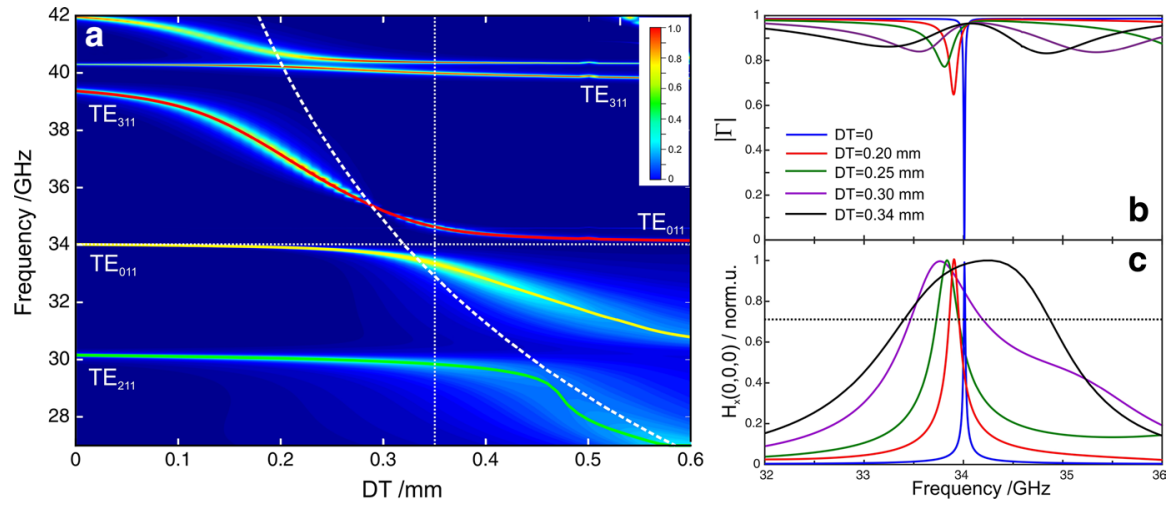

Fig. 4 a Dependence of the reflection coefficient, $|\Gamma|$ on the dielectric plate thickness, shown as a contour plot, calculated for a circular iris diameter of $2.6 \mathrm{~mm}$ and short position of $L=-10.6 \mathrm{~mm}$. The solid lines show the dependencies of cavity resonances below $42 \mathrm{GHz}$. The dashed curve shows the calculated resonance frequency of the circular iris in a WR28 waveguide. The horizontal dotted line indicates the $\mathrm{TE}_{011}$ mode resonance frequency of $34 \mathrm{GHz}$ at DT $=0 \mathrm{~mm}$. The vertical dotted line indicates the dielectric thickness DT $=0.34 \mathrm{~mm}$, i.e., the point that most closely approaches the resonance modes indicated by red and yellow solid lines. b Reflection coefficient and $\mathbf{c} x$-component of the magnetic field amplitude at the resonator center for a cavity with circular iris of $2.6 \mathrm{~mm}$ calculated for the critically coupled case without a dielectric plate, and for the maximally coupled case with dielectric plates of different thicknesses 
analyzed. The reflection coefficient and $x$-component of the magnetic field amplitude were calculated at selected thicknesses of the dielectric plate between 0.20 and $0.34 \mathrm{~mm}$ and the results are shown in Fig. 4b, c and summarized in Table 2. The key observation to be made here is that the reduction in the maximum magnetic field amplitude at the sample position is lower than expected. The calculation for $\mathrm{DT}=0.34 \mathrm{~mm}$ yields a reduction factor of 6.2 , which is smaller compared to the factor calculated from bandwidths assuming a "true" overcoupling regime, i.e., 7.3; see Eq. (1) and SI. Thus, the mode mixing described above is advantageous, acting to concentrate the magnetic field in the region of space occupied by the sample.

\subsection{Experimental Results}

The reliability of the electromagnetic simulations described above was tested experimentally. Cavity configurations, both without a dielectric plate and with plates of different thickness fixed at the iris, were investigated at room temperature and low temperature $(20 \mathrm{~K})$. A comparison of the cavity ringdown measurements and microwave nutation experiments with the electromagnetic simulations are shown in Fig. 5, and summarized in Table 3 . The simulation parameters were adjusted to match the properties and dimensions of the real probehead, e.g., the iris diameter was reduced to $2.5 \mathrm{~mm}$ in accordance with the real cavity.

The parameters for a critically coupled cavity without a dielectric plate at room temperature are used as the reference parameters. A cavity bandwidth of $13.8 \pm 0.6 \mathrm{MHz}$ was evaluated from the ringdown trace and frequency dependence of the microwave nutation frequency (Fig. 4). This value is in a good agreement with the calculated value of $13.9 \mathrm{MHz}$, verifying the model parameters. The amplitude of the microwave magnetic field can be compared using the length of the $\pi$-pulse, $t_{p}(\pi)$, which is directly evaluated from the microwave nutation traces at

Table 2 Parameters of cavity models for different thicknesses of dielectric plate

\begin{tabular}{|c|c|c|c|c|c|c|}
\hline \multirow[t]{2}{*}{$\mathrm{DT} / \mathrm{mm}$} & \multicolumn{2}{|c|}{$\nu_{0}^{\mathrm{a}} / \mathrm{GHz}$} & \multicolumn{2}{|c|}{$\Delta \nu_{1 / 2} / \mathrm{MHz}^{\mathrm{b}}$} & \multicolumn{2}{|c|}{$\begin{array}{l}H_{x} \\
(0,0,0)^{\mathrm{e}} / \frac{\mathrm{A}}{\mathrm{m}} \cdot \frac{1}{\sqrt{\mathrm{W}}} \\
\end{array}$} \\
\hline & $\beta=1^{c}$ & $\max . \beta^{\mathrm{d}}$ & $\beta=1$ & $\max . \beta$ & $\beta=1$ & $\max . \beta$ \\
\hline 0 & 34.018 & 34.014 & 13.7 & 18.7 & 909 & 872 \\
\hline 0.20 & 33.914 & 33.910 & 19.6 & 96.1 & 769 & 456 \\
\hline 0.25 & 33.832 & 33.841 & 26.4 & 206 & 640 & 310 \\
\hline 0.30 & 33.506 & 33.773 & 59.2 & 534 & 343 & 193 \\
\hline 0.34 & $\begin{array}{l}33.133 \\
34.629\end{array}$ & 34.286 & $\begin{array}{l}80.1 \\
57.7\end{array}$ & 1450 & $\begin{array}{l}203 \\
342\end{array}$ & 146 \\
\hline
\end{tabular}

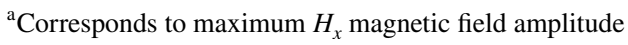

${ }^{\mathrm{b}}$ Determined from the frequency dependence of $H_{x}$

${ }^{\mathrm{c}}$ The short position adjusted for critical coupling

${ }^{\mathrm{d}}$ The short position adjusted for maximum overcoupling

${ }^{\text {e}}$ The maximum $H_{x}$ is obtained in the geometrical center of the cavity. The magnetic field distributions over the sample volume are the same for all situations within $2 \%$ 

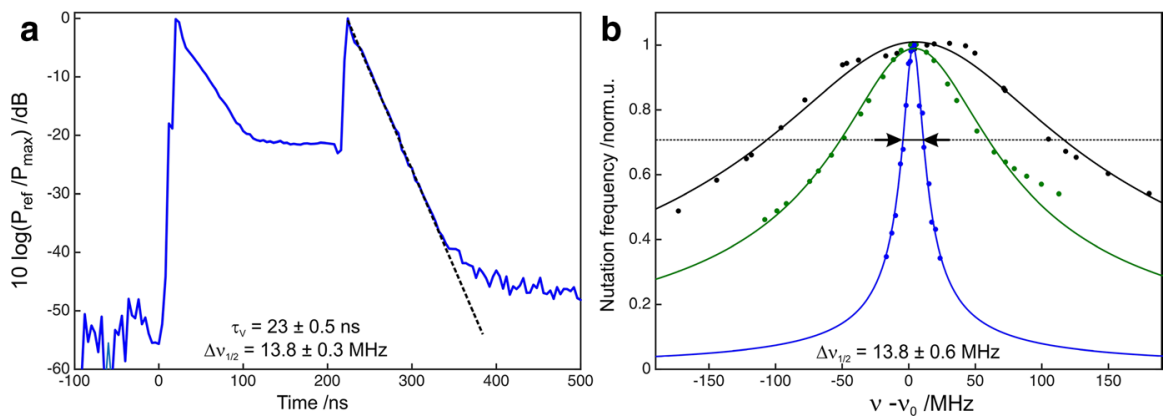

Fig. 5 a Experimental mw power profile recorded for a cavity without a dielectric plate, applying a mw pulse of $200 \mathrm{~ns}$ at room temperature. The flat part during the pulse yields a return loss of $20 \mathrm{~dB}$ $(|\Gamma|=0.1)$. The linear approximation of the power decay after the mw pulse is switched off (dashed line) yields a power decay time of $11.5 \pm 0.2 \mathrm{~ns}$ (voltage ringing time $\tau_{V}=23 \pm 0.5 \mathrm{~ns}$ ), which corresponds to a bandwidth of $\Delta v_{1 / 2}=\frac{1}{\pi \tau_{V}}=13.8 \pm 0.3 \mathrm{MHz}$. b Frequency dependence of the relative microwave nutation frequencies at room temperature for (i) a critically coupled cavity without a dielectric plate (blue dots) and (ii) for a maximally overcoupled cavity with a dielectric plate of DT $=0.25 \mathrm{~mm}$ (green dots) and $0.29 \mathrm{~mm}$ (black dots). The lines show the best fit result of the experimental data to the square root of a Lorentzian function. The dashed line indicates the level of 0.707 . The absolute values of the $\pi$-pulse length obtained at the maximum evaluable mw power are summarized in Table 3

Table 3 Experimental and calculated cavity parameters

\begin{tabular}{|c|c|c|c|c|c|c|}
\hline \multicolumn{2}{|c|}{$\mathrm{DT} / \mathrm{mm}$} & \multicolumn{2}{|c|}{$\Delta \nu_{1 / 2} / \mathrm{MHz}$} & \multicolumn{2}{|c|}{$\mathrm{t}_{p}(\pi) / \mathrm{ns}$} & \multirow{2}{*}{$\frac{t_{p}(\max . \beta)}{t_{p}(D T=0, \beta=1)}$} \\
\hline & & $\beta=1$ & $\max . \beta$ & $\beta=1$ & $\max . \beta$ & \\
\hline \multirow[t]{2}{*}{0} & calc & 13.9 & - & $10.0^{a}$ & & 1 \\
\hline & $\exp$ & $\begin{array}{l}13.8 \\
(12.7)^{\mathrm{c}}\end{array}$ & - & $\begin{array}{l}15.6^{\mathrm{b}} \\
(14.3)^{\mathrm{c}}\end{array}$ & & 1 \\
\hline \multirow[t]{2}{*}{0.21} & calc & 16.6 & 75 & 11.4 & 17.7 & 1.8 \\
\hline & $\exp$ & 21.0 & 59 & 19.2 & 22.7 & 1.4 \\
\hline \multirow[t]{2}{*}{0.25} & calc & 21.8 & 132 & 14.2 & 23.3 & 2.4 \\
\hline & exp & $\begin{array}{l}41.7 \\
(52.7)^{\mathrm{c}}\end{array}$ & $\begin{array}{l}97 \\
(124)^{\mathrm{c}}\end{array}$ & 27.8 & $\begin{array}{l}37.0 \\
(43.0)^{\mathrm{c}}\end{array}$ & 2.2 \\
\hline \multirow[t]{2}{*}{0.29} & calc & 36.9 & 275 & 18.5 & $33.5^{\mathrm{d}}$ & 3.4 \\
\hline & $\exp$ & 77.1 & 219 & 38.4 & 48.6 & 3.0 \\
\hline
\end{tabular}

${ }^{a}$ Calculated from the simulated magnetic field amplitude for $10 \mathrm{~W}$ mw power on cavity

${ }^{\mathrm{b}}$ Evaluated from experimental nutation recordings at full mw power ${ }^{\mathrm{c}}$ At $20 \mathrm{~K}$ (see SI)

${ }^{\mathrm{d}}$ Corresponds to $t_{p}(\pi)=8.6 \mathrm{~ns}$ for $150 \mathrm{~W}$ TWT amplifier

cavity resonance. A value of $t_{p}(\pi)=15.6 \pm 1 \mathrm{~ns}$ was easily achieved at the full microwave power (see SI for bridge outputs). The maximum mw magnetic field amplitude, $H_{x}$, is converted to the expected pulse length as $t_{p}(\pi)=\frac{2 \pi}{\sqrt{W} \mu_{0} \gamma_{e} H_{x}}$, with $W, \mu_{0}$ and $\gamma_{e}$ being the mw power at the cavity, permeability of a vacuum, and electron gyromagnetic ratio respectively. The theoretical value of $t_{p}(\pi)$ is $10 \mathrm{~ns}$ 
$\left(H_{x}(0,0,0)=890 \frac{\mathrm{A}}{\mathrm{m}} \cdot \frac{1}{\sqrt{\mathrm{W}}}\right.$ and $\left.W=10 \mathrm{~W}\right)$, which is about a factor of 1.5 lower than the experimental one. This discrepancy probably stems from the overestimation of mw power at the cavity which cannot be exactly determined experimentally. Therefore, for subsequent comparisons, we used the experimental and calculated pulse length normalized to the pulse length for a critically coupled cavity without a dielectric plate (Table 3).

Good agreement is seen between the calculated and experimental values in the overcoupling regime. Calculations predict slightly higher bandwidths at maximum overcoupling, which is most likely due to overestimation of the dielectric constant (Rogers 3010 material, $\varepsilon_{\mathrm{r}}=10.7$ ). If a slightly lower value (10.4) is used, which lies within the manufacturer's specifications, a better match between calculation and experiment is obtained. The behavior of the magnetic field amplitude, $t_{p}(\pi)$, perfectly agrees with the behavior of the cavity bandwidth, see Table 3 .

Agreement is less good between calculated and experimental bandwidths (and magnetic field amplitudes) in the critically coupled regime, which increases as a function of the dielectric thickness. This is likely due to the Rogers laminate material having a significantly higher dielectric loss factor than assumed. The calculation uses the loss tangent specified at $10 \mathrm{GHz}\left(\tan \delta=2.3 \cdot 10^{-3}\right)$. There is no reliable data for $\tan \delta$ at $34 \mathrm{GHz}$ and recent studies have shown that Rogers laminate materials show significantly higher dielectric loss factors at high frequencies than those specified at lower frequencies $(<10 \mathrm{GHz})$ [11]. If the loss factor is increased to $\tan \delta=10^{-2}$, the calculated bandwidths for the critically coupled cavity are in good agreement with the experimental values.

\subsection{Coupling Mechanism}

The experimental and simulation results demonstrate that the cavity bandwidth can be increased without compromising the pulsed EPR performance; the resonance properties of the circular iris can be "tuned" using a dielectric material positioned at the front of the iris. While the dielectric plate coupling scheme allows for large, tunable overcoupling and therefore a large bandwidth, the use of a fixed plate has several disadvantages in the context of a general purpose cavity. Firstly, the resonance properties of the iris become fixed, which leads to the fixed, distortedmode profile in the cylindrical cavity described above. The mixed-mode character results in higher losses in the cavity, reducing the amplitude of the microwave magnetic field at the sample position, which diminishes its performance in CW EPR experiments. Secondly, the very high iris transmittance requires precise adjustment of the short position to accurately set the critical coupling position. This leads to the cavity coupling being highly sensitive to mechanical vibrations and pressure variations in the cryostat, again compromising the resonator performance for $\mathrm{CW}$ EPR.

To overcome these limitations, we propose a coupling mechanism in which the dielectric plate is fixed onto the movable short, rather than onto the iris. The waveguide-cavity transmission has a periodic dependence on the distance of the short 
from the iris (Fig. 2c and Fig. S02 in the SI); hence, a short-fixed dielectric plate of sufficient length should allow for two interchangeable regimes of operation. These regimes are illustrated by the simulations in Fig. 6, calculated for a dielectric plate of $10 \mathrm{~mm}$ length (DT $=0.3 \mathrm{~mm}$ ) attached to the movable short. Positioning the short between $-14 \mathrm{~mm}$ to $-11 \mathrm{~mm}$ from the iris center would allow the coupling of the waveguide-iris-cavity configuration to be varied without influence from the dielectric plate, i.e., without the dielectric plate changing the iris properties. This first regime does not allow for overcoupling, but is advantageous for critical coupling of a high-Q cavity. The dielectric plate starts to interact with the iris and increases the iris transmittance from short positions above $-11 \mathrm{~mm}$. This second regime is suitable for controlled overcoupling of the cavity, as required for pulse EPR experiments. This position can also be used for critically coupling a cavity that is loaded with a sample with substantial dielectric losses. In this case, the power losses from the sample will overcome the power losses caused by the dielectric plate, and mode distortions can be neglected.

Experimental results up to this point suggest that dielectric laminates are not an ideal material for the dielectric coupling plate. Their dielectric constant is strongly temperature-dependent and the laminate material displays poor mechanical properties. Ongoing work of our laboratories is the manufacture of a new short coupler made from sapphire. Sapphire has good mechanical properties
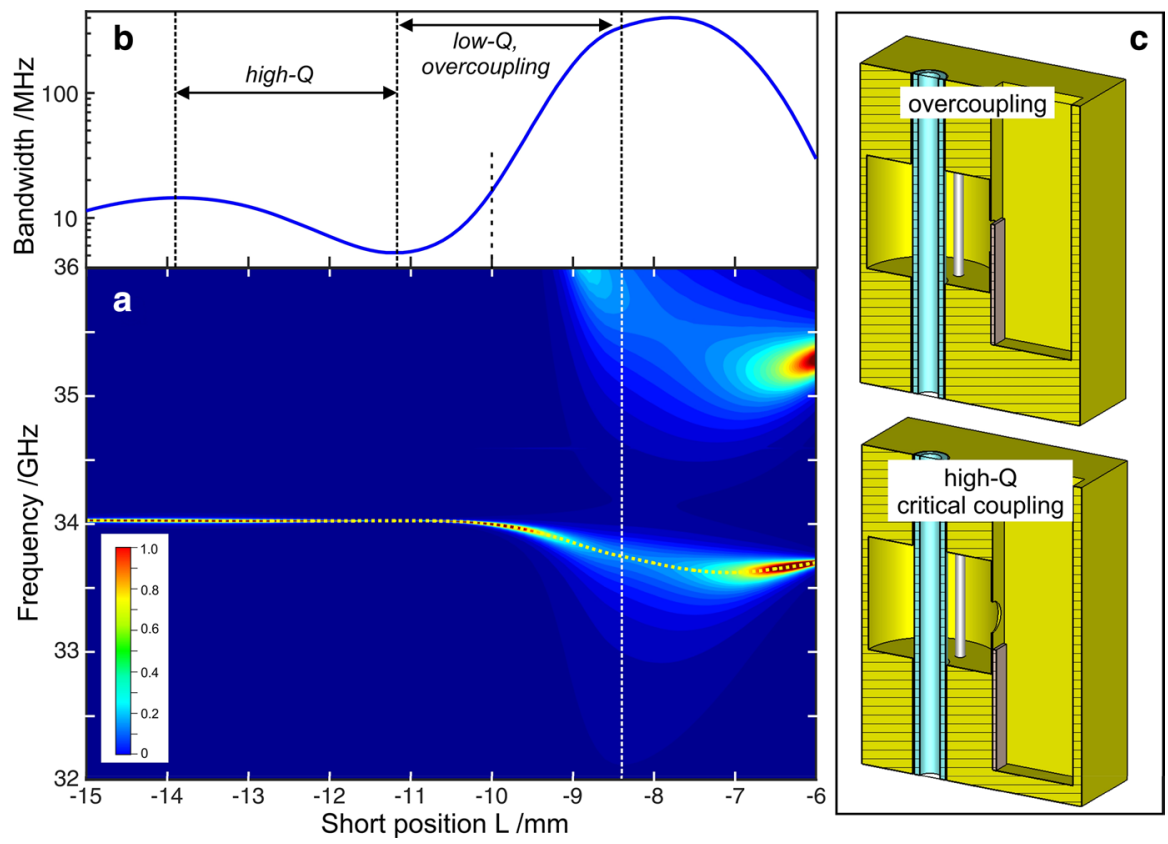

Fig. 6 a Dependence of the reflection coefficient, $|\Gamma|$ on the position of the short carrying the dielectric plate $(\mathrm{DT}=0.3 \mathrm{~mm})$ of $10 \mathrm{~mm}$ length. The yellow dashed line shows the behavior of the cavity operation mode. b The cavity bandwidth evaluated from $S_{11}$ curves. Two regimes of operation are indicated and visualized in (c) 
and, importantly, low dielectric losses $\left(\tan \delta \sim 10^{-5}\right.$ at room temperature, $\sim 10^{-7}$ at temperatures below $100 \mathrm{~K}$ ) [12] such that they can be safely neglected. The choice of crystal cut allows us to choose between two temperature-stable dielectric constants (9.4 and 11.6 at room temperature) [12].

\subsection{Alternative Iris Geometries}

This work has so far only considered a circular iris. However, the original description of the $\mathrm{TE}_{011}$ cavity published in [5] used a slot-type iris. This raises the question: does the slot-type iris pose any advantages over a circular iris? The slot-type iris has the "native" geometry for the $\mathrm{TE}_{01}$ wave transfer in a rectangular waveguide. The self-resonance of the slot can be tuned to a particular frequency by choosing the slot geometry. The resonance frequency of the narrow rectangular slot is approximately equal to the cutoff frequency of a waveguide having the same cross-sectional shape as the aperture [13]. Thus, an empirical relationship for a resonant slot of height, $h$, and width $w$, centered in the transverse plane of a rectangular waveguide for small $w / h$, reduces to $h \approx \lambda_{0} / 2$. For a narrow rounded slot, some correction has to be made, $h \approx \frac{\lambda_{0}}{2}+0.273 \cdot w[14]$. This results in a rounded slot height of $4.55 \mathrm{~mm}$ for a self-resonance frequency at $34 \mathrm{GHz}$. Simulations predict an optimal height of $h=4.6 \mathrm{~mm}$ for $w=0.5 \mathrm{~mm}$, which closely matches the above estimation; see Figs. S7 in the SI. The maximum iris transmission coefficient is very high, $|T|=1$. However, the transmission curve of the isolated resonant slot is very broad, giving a bandwidth of $14.3 \mathrm{GHz}$, which efficiently couples at least all three fundamental modes, $\mathrm{TE}_{211}, \mathrm{TE}_{011}$ and $\mathrm{TE}_{311}$. This results in unwanted effects for the critically coupled cavity, i.e., the maximum magnetic field amplitude in mixed modes, and $H_{x}$, reduces by about a factor of 3 as compared to the small round aperture coupling. A non-resonant rounded slot of $h w=3.0 \times 0.5 \mathrm{~mm}^{2}$ on the other hand, has the same transmittance at $34 \mathrm{GHz}$ as compared to the circular iris of $2.6 \mathrm{~mm}$ diameter (Fig. S9 in SI). The self-resonance frequency of this slot is about $50.8 \mathrm{GHz}$, but this frequency can also be successfully downshifted by means of a dielectric plate. There are, however, two crucial differences between the $3.0 \times 0.5 \mathrm{~mm}^{2}$ slot iris and its $2.6 \mathrm{~mm}$ circular counterpart. Firstly, due to the higher electric field in the slot iris (higher capacitance), it requires significantly less dielectric (Fig. S8). For an isolated $3.0 \times 0.5 \mathrm{~mm}^{2}$ slot iris, a dielectric plate of only $0.098 \mathrm{~mm}$ thickness will match the self-resonance frequency to $34 \mathrm{GHz}$. This problem, however, can be avoided by using dielectric materials with smaller dielectric constant. Secondly, the transmission bandwidth for the slot iris is $4.6 \mathrm{GHz}$, almost two times smaller than the $8.7 \mathrm{GHz}$ transmission bandwidth of the circular iris. Additionally, the transmission properties of the slot iris were found to be more critically dependent on system parameters such as geometry of the slot, iris wall thickness, geometry of the dielectric plate and its dielectric constant. In practice, this means that a real slot iris coupling system would be less predictable than the circular iris. A significant advantage of the circular iris is also the simplicity and achievable precision of its fabrication, especially when extending the applicability of the coupling mechanism to higher microwave frequencies. At $95 \mathrm{GHz}$ (W-band) or even $263 \mathrm{GHz}$, the 
circular iris of $0.9 \mathrm{~mm}$ diameter or $0.33 \mathrm{~mm}$, respectively can be easily machined mechanically. The precise manufacturing of the slots $\left(1.07 \times 0.18 \mathrm{~mm}^{2}\right.$ for $95 \mathrm{GHz}$, $0.39 \times 0.06 \mathrm{~mm}^{2}$ for $263 \mathrm{GHz}$, which are scaled from the Q-band slot dimensions) becomes challenging for mechanical machining and would require more advanced fabrication techniques.

\section{Conclusions}

We have demonstrated that the resonance properties of the iris can be effectively manipulated using dielectric plates. The modifications to the general purpose Q-band probehead described in this work allow the precise adjustment of the iris' transmission toward the $\mathrm{TE}_{011}$ cavity, and enable the construction of new types of coupling mechanisms. The new mechanism is based on the combined action of a short-type coupler and dielectric plate coupler, and operates in two regimes which allow both critical coupling of a high-Q cavity without diminishing CW EPR performance, as well as large cavity overcoupling to $>1 \mathrm{GHz}$ bandwidths. This greatly expands the versatility of the original probehead, now fulfilling the bandwidth requirements for a wide range of different EPR experiments including CW EPR, pulse EPR, pulse ENDOR and DEER.

The possibility to manipulate the resonance properties of different iris types opens further directions for the development of new coupling mechanisms for different types of cavities. We have already developed such a mechanisms for end waveguide to cavity configurations, e.g., Fabry-Perot Q-band, cylindrical $\mathrm{TE}_{011} \mathrm{~W}$-band and rectangular $\mathrm{TE}_{102} \mathrm{X}$-band cavities. At high microwave frequencies in particular, e.g., W-band and above, such coupling mechanisms allow for more flexible cavity applications compared to other types of iris-based couplings such as those described in [15-18]. We plan to describe these findings in forthcoming publications.

Supplementary Information The online version contains supplementary material available at https://oi. org/10.1007/s00723-021-01404-4.

Acknowledgements We are grateful to Klaus Möbius and Kev Salikhov for their inspiring research and support. We congratulate them on their 85th birthday and wish them all the best for the years to come. N.C. acknowledges the support of the Australian Research Council (DP210100088).

Funding Open Access funding enabled and organized by Projekt DEAL.

Open Access This article is licensed under a Creative Commons Attribution 4.0 International License, which permits use, sharing, adaptation, distribution and reproduction in any medium or format, as long as you give appropriate credit to the original author(s) and the source, provide a link to the Creative Commons licence, and indicate if changes were made. The images or other third party material in this article are included in the article's Creative Commons licence, unless indicated otherwise in a credit line to the material. If material is not included in the article's Creative Commons licence and your intended use is not permitted by statutory regulation or exceeds the permitted use, you will need to obtain permission directly from the copyright holder. To view a copy of this licence, visit http://creativeco mmons.org/licenses/by/4.0/. 


\section{References}

1. N. Cox, A. Nalepa, M.-E. Pandelia, W. Lubitz, A. Savitsky, Chapter Nine - Pulse Double-Resonance EPR Techniques for the Study of Metallobiomolecules. In Methods in Enzymology (Qin Peter, Z. and W. Kurt, eds.). Academic Press, pp. 211-249 (2015)

2. N. Cox, W. Lubitz, A. Savitsky, Mol. Phys. 111, 2788-2808 (2013)

3. A. Schweiger, G. Jeschke, Principles of Pulse Electron Paramagnetic Resonance (Oxford University Press, Oxford, 2001)

4. C. Fainstein, S.B. Oseroff, Rev. Sci. Instr. 42, 547-548 (1971)

5. E. Reijerse, F. Lendzian, R. Isaacson, W. Lubitz, J. Magn. Reson. 214, 237-243 (2012)

6. A. Sienkiewicz, B.G. Smith, A. Veselov, C.P. Scholes, Rev. Sci. Instr. 67, 2134-2138 (1996)

7. Y. Ohba, C. Watanabe, S. Nakazawa, S. Yamauchi, Appl. Magn. Reson. 37, 781 (2009)

8. I. Gromov, J. Forrer, A. Schweiger, Rev. Sci. Instr. 77, 064704 (2006)

9. R.R. Mett, J.W. Sidabras, J.S. Hyde, Appl. Magn. Reson. 35, 285-318 (2009)

10. G.A. Rinard, R.W. Quine, S.S. Eaton, G.R. Eaton, W. Froncisz, J. Magn. Reson. Ser. A. 108, 71-81 (1994)

11. J.A. Hejase, P.R. Paladhi, P.P. Chahal, IEEE Trans. Compon. Pack. Manuf. Technol. 1, 1685-1694 (2011)

12. J. Krupka, K. Derzakowski, M. Tobar, J. Hartnett, R.G. Geyer, Meas. Sci. Technol. 10, 387-392 (1999)

13. J.C. Slater, Microwave Transmission (McGraw-Hill Book Company, New York, 1942)

14. S.B. Cohn, Proc. IRE. 40, 696-699 (1952)

15. A. Savitsky, Y. Grishin, R. Rakhmatullin, E. Reijerse, W. Lubitz, Rev. Sci. Instr. 84, 014704 (2013)

16. O. Burghaus, M. Rohrer, T. Götzinger, M. Plato, K. Möbius, Meas. Sci. Technol. 3, 765-774 (1992)

17. H. Blok, J.A.J.M. Disselhorst, S.B. Orlinskii, J. Schmidt, J. Magn. Reson. 166, 92-99 (2004)

18. J.A.J.M. Disselhorst, H. van der Meer, O.G. Poluektov, J. Schmidt, J. Magn. Reson. A. 115, 183188 (1995)

Publisher's Note Springer Nature remains neutral with regard to jurisdictional claims in published maps and institutional affiliations. 